Poster 279

Radial Neuropathy Exacerbated by Ligamentous Laxity in Pregnancy and by Chemotherapy: A Case Report

Joel Castellanos, MD (University of Michigan, Ypsilanti, Michigan, United States), Sean R. Smith, MD

Disclosures: Joel Castellanos: I Have No Relevant Financial Relationships To Disclose

Case/Program Description: A 32-year-old right-handed woman with metastatic malignant mixed Mullerian tumor (MMMT) presented with left upper extremity numbness, tingling, and pain along her posterior forearm, thumb, and second digit. She developed mild symptoms during pregnancy 7 months prior, and severe symptoms three months before presentation after receiving carboplatin and paclitaxel chemotherapy for MMMT. Four years earlier, she also experienced a milder form of these symptoms; electromyography (EMG) found no abnormalities and the symptoms resolved spontaneously. Of note, her history is significant for numerous left shoulder subluxations requiring reduction.

Setting: Outpatient cancer rehabilitation clinic.

Results: Physical examination revealed decreased strength in left elbow extension (4-/5) and wrist extension (4/5), absent left triceps reflex, and diminished pinprick sensation over the posterior forearm with increased sensitivity to light touch over the distal radial nerve distribution. Computerized tomography of her neck and chest did not reveal neural foraminal narrowing or metastatic disease to explain her symptoms. On EMG testing, radial sensory nerve conduction studies (NCS) were not recordable, and radial motor studies at the forearm showed decreased amplitude compared to the right side $(1.7 \mathrm{mV}$ vs $3.7 \mathrm{mV})$ with comparable distal latencies. Median and ulnar motor and sensory NCS were normal. Needle electromyography revealed positive waves, fibrillations, and chronic changes in the extensor carpi radialis brevis, brachioradialis, and extensor indicis, but not the triceps, suggesting an incomplete chronic axonal neuropathy proximal to the brachioradialis. Deltoid, biceps brachii, pronator teres, and first dorsal interosseus (hand) had no abnormal EMG findings.

Discussion: Repetitive shoulder subluxation likely created tension on the radial nerve at the spiral groove of the humerus, causing a stretch injury. Ligamentous laxity in pregnancy caused symptoms to recur, and chemotherapy worsened the injury to the point of axonotmesis.

Conclusions: Pregnancy may exacerbate neuropathies associated with ligamentous laxity, and chemotherapy can worsen pre-existing neuropathies.

Level of Evidence: Level $\mathrm{V}$

\section{Poster 280}

ALS Masked by Breast Cancer History and Low Back Pain: A Case Report

Patrick Dolan, MD (SUNY Downstate Medical Center, Brooklyn, NY, United States), Bhavi Patel, DO, Marcel G. Bayol, MD

Disclosures: Patrick Dolan: I Have No Relevant Financial Relationships To Disclose

Case/Program Description: A 56-year-old African American woman with a past medical history of hypertension and ductal carcinoma in situ of the breast (treated by resection and radiation) was evaluated for worsening leg and arm weakness. She had leg weakness requiring the use of a cane. She later developed arm weakness resulting in decreased grip with her cane and almost daily falls. She also noted low back pain and occasional urinary incontinence. There was concern for metastases or a paraneoplastic syndrome from her breast cancer history but CT and MRI of the head revealed no new changes. CT of the spine revealed L3-S1 disk herniations (L3/4, L4/5, L5/S1) so she was given two epidural steroid injections for possible lumbar radiculopathy. Her symptoms continued to worsen with new vocal hoarseness accompanied by shortness of breath. On initial exam, she was found to have 4/5 muscle strength with $3+$ hyperreflexia throughout, upgoing plantar reflex bilaterally and a positive jaw jerk reflex. She was able to transfer independently and exhibited a very slow and narrow, unsteady gait. Otherwise, the rest of her examination was within normal limits. Workup including antibodies, inflammatory markers, and infectious etiologies were all negative except for a CK of 777. MRI of spine corroborated previous CT findings. After no imaging or laboratory abnormalities pointed to any diagnosis, EMG was performed leading to Amyotrophic Lateral Sclerosis (ALS) diagnosis. Patient was subsequently transferred to acute inpatient rehab then referred to outpatient movement disorder specialist for further care.

Setting: Inpatient Acute Rehabilitation Facility.

Results: EMG demonstrated reduced amplitude, prolonged latencies, or decreased conduction velocity in right median and ulnar motor nerves, right medial sensory nerve, right peroneal motor and sensory nerves, and right sural sensory nerve.

Discussion: This case demonstrates the importance of physical exam and significance of inpatient electrodiagnostic studies in helping to establish or confirm a diagnosis.

Conclusions: Based on our physical examination revealing UMN/LMN signs with $L M N$ findings corroborated by EMG and absence of other more likely diagnoses this patient demonstrates ALS.

Level of Evidence: Level $\mathrm{V}$

\section{Poster 281}

The Adult Spasticity International Registry (ASPIRE

Study): Baseline Demographics and Clinical Characteristics of Patients Treated for Spasticity

Gerard E. Francisco, MD (University of Texas and TIRR Memorial, Houston, TX, United States), Daniel Bandari, Ganesh Bavikatte, Wolfgang H. Jost, MD, PhD, Aubrey Manack Adams, PhD, Joan Largent, Alberto Esquenazi, MD

Disclosures: Gerard Francisco: Research Grants - Allergan, Research Grants - Merz, Research Grants - Mallinckrodt, Research Grants - Ipsen, Research Grants - Nexstim, Research Grants - Micro transponder Objective: To describe baseline demographics and clinical characteristics in patients treated with onabotulinumtoxinA for spasticity. Design: Multicenter, prospective, observational registry study (NCT01930786).

Setting: Select clinical sites in North America, Europe, and Asia. Participants: Patients with spasticity of multiple etiologies, including those naïve to and previously treated with onabotulinumtoxinA. Interventions: OnabotulinumtoxinA treatments administered per routine care.

Main Outcome Measures: Primary objectives were to assess onabotulinumtoxinA treatment utilization, patient/physician treatment satisfaction, and botulinum toxin treatment incidence for other indications among adult spasticity patients treated with onabotulinumtoxinA. Baseline demographics and clinical characteristics as of September 10, 2015 are summarized (enrollment completed September 11, 2015).

Results: A total of 727 patients were enrolled in ASPIRE and treated by 63 healthcare providers (69.4\% PM\&R's) across France, Germany, Italy, Spain, Taiwan, UK, and USA ( $\mathrm{n}=54$ sites). More patients $(\mathrm{n}=602$ / $715,84.2 \%$ ) had lower limb spasticity (most common: equinovarus foot [35.2\%] and extended knee [16.1\%]), than upper limb spasticity $(\mathrm{n}=513 / 716,71.2 \%$; most common: flexed elbow [22.4\%] and clenched fist [21.5\%]). At baseline, $36.8 \%(n=268)$ of patients were botulinum toxin naïve for spasticity treatment. Patients with spasticity due to stroke $(58.1 \%)$, multiple sclerosis $(15.7 \%)$, other etiologies $(10.1 \%)$, cerebral palsy $(9.8 \%)$, traumatic brain injury $(5.9 \%)$, and spinal cord injury (5.4\%), were on average 53.7 years old, majority were Caucasian (77.4\%), and gender was nearly evenly distributed ( $52.1 \%$ female). Patients were mainly on disability $(44.2 \%)$ and had a caregiver (57.9\%; 